\title{
The third law of thermodynamics in open quantum systems
}

Cite as: J. Chem. Phys. 151, 064115 (2019); https://doi.org/10.1063/1.5100182

Submitted: 15 April 2019 . Accepted: 21 July 2019 . Published Online: 13 August 2019

Abhay Shastry, Yiheng Xu, and Charles A. Stafford (D)

\section{COLLECTIONS}

Paper published as part of the special topic on Dynamics of Open Quantum Systems Note: This paper is part of a JCP Special Topic on Dynamics of Open Quantum Systems.
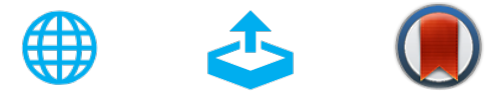

\section{ARTICLES YOU MAY BE INTERESTED IN}

Large deviations and dynamical phase transitions in stochastic chemical networks The Journal of Chemical Physics 151, 064117 (2019); https://doi.org/10.1063/1.5111110

Ions' motion in water

The Journal of Chemical Physics 150, 190901 (2019); https://doi.org/10.1063/1.5090765

Similarity transformation of the electronic Schrödinger equation via Jastrow factorization The Journal of Chemical Physics 151, 061101 (2019); https://doi.org/10.1063/1.5116024

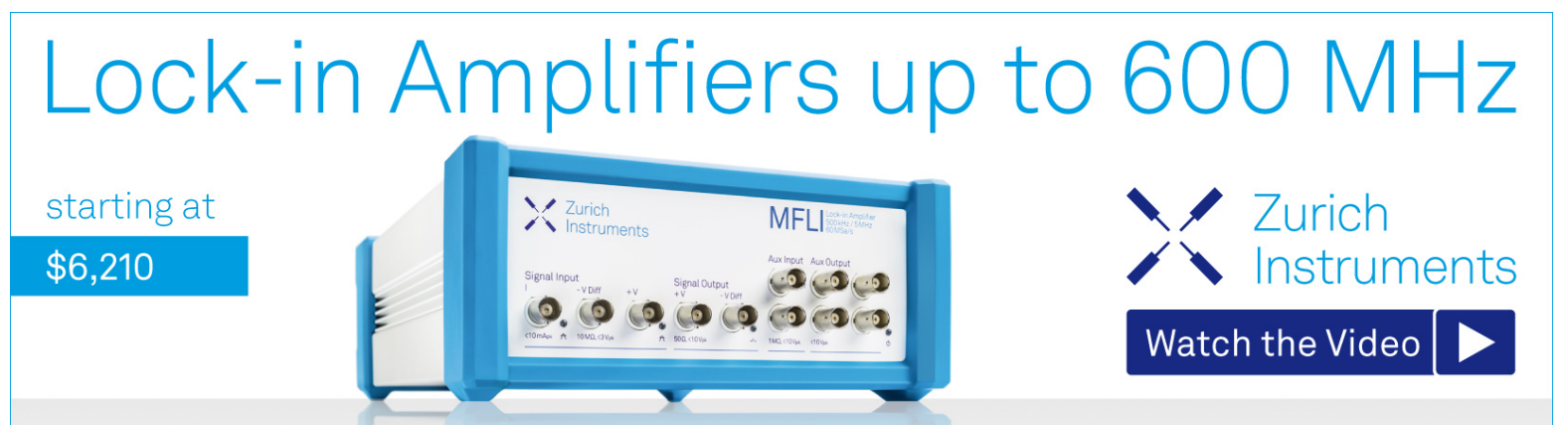




\title{
The third law of thermodynamics in open quantum systems
}

\author{
Cite as: J. Chem. Phys. 151, 064115 (2019); doi: 10.1063/1.5100182 \\ Submitted: 15 April 2019 Accepted: 21 July 2019 • \\ Published Online: 13 August 2019
}

\begin{abstract}
Abhay Shastry, ${ }^{1,2, a)}$ Yiheng $\mathrm{Xu},{ }^{1,3, a)}$ and Charles A. Stafford
AFFILIATIONS

${ }^{1}$ Department of Physics, University of Arizona, 1118 East Fourth Street, Tucson, Arizona 85721, USA

${ }^{2}$ Department of Chemistry, University of Toronto, 80 St. George Street, Toronto, Ontario M5S 3H4, Canada

${ }^{3}$ Department of Physics, University of California, San Diego, California 92093, USA
\end{abstract}

Note: This paper is part of a JCP Special Topic on Dynamics of Open Quantum Systems.

a) Contributions: A. Shastry and Y. Xu contributed equally to this work.

\begin{abstract}
We consider open quantum systems consisting of a finite system of independent fermions with arbitrary Hamiltonian coupled to one or more equilibrium fermion reservoirs (which need not be in equilibrium with each other). A strong form of the third law of thermodynamics, $S(T) \rightarrow 0$ as $T \rightarrow 0$, is proven for fully open quantum systems in thermal equilibrium with their environment, defined as systems where all states are broadened due to environmental coupling. For generic open quantum systems, it is shown that $S(T) \rightarrow g \ln 2$ as $T \rightarrow 0$, where $g$ is the number of localized states lying exactly at the chemical potential of the reservoir. For driven open quantum systems in a nonequilibrium steady state, it is shown that the local entropy $S(\mathbf{x} ; T) \rightarrow 0$ as $T(\mathbf{x}) \rightarrow 0$, except for cases of measure zero arising due to localized states, where $T(\mathbf{x})$ is the temperature measured by a local thermometer.
\end{abstract}

Published under license by AIP Publishing. https://doi.org/10.1063/1.5100182

\section{INTRODUCTION}

There are two formulations of the third law of thermodynamics, both due to Nernst: (A) the Nernst heat theorem, which states that the equilibrium entropy of a pure substance goes to zero at zero temperature, and (B) the unattainability principle, which states that it is impossible to cool any system to absolute zero in a finite number of operations.

The third law in open quantum systems has been discussed in various contexts. Kosloff and collaborators ${ }^{1}$ consider the unattainability principle (B) in the context of quantum absorption refrigerators and show that it is not possible to cool to absolute zero in finite time. The authors warn that the quantum master equation has to be used carefully and that violations of the laws of thermodynamics could result otherwise. The unattainability principle was challenged in Ref. 2, with claims that zero temperature can be reached but that formulation (A) still holds true. Reference 3 arrives at a result which is in violation of the unattainability principle as pointed out by a comment by Kosloff. ${ }^{4}$ Kosloff generally advocates for a careful use of the master equation and in Ref. 5 argues that such apparent violations ${ }^{2}$ of the laws of thermodynamics are caused by uncontrolled approximations. Reference 6 provides a proof of the unattainability principle (B) using quantum resource theory and clarifies its connection to the heat theorem (A).

Statement (A) of the third law was proven for a quantum oscillator in contact with various types of heat baths in Refs. 7 and 8 . In Ref. 8, O'Connell rebuts the early claims of the violations of the laws of thermodynamics made in the field of quantum thermodynamics. In particular, he focuses on Ref. 9, which claims to construct a perpetual motion machine. As related to the third law, Ref. 9 argues that "neither the von Neumann entropy nor the Boltzmann entropy vanishes when the bath temperature is zero," leading to a claim of a violation of the third law for nonweak coupling. O'Connell calculates the von Neumann entropy ${ }^{8}$ and points out that when the interaction energy is considerable, the von Neumann formula can only be applied to the entire system and not to the reduced system.

Perhaps, the most flagrant violation of the third law of thermodynamics was put forward by Esposito, Ochoa, and Galperin, ${ }^{10}$ who claim not only that Nernst's heat theorem does not hold, but that 
the entropy of an open quantum system is undefined in the limit of zero temperature. Their approach is inspired by that of Sanchez and co-workers, ${ }^{11,12}$ who argue that the definition of heat in open quantum systems is ambiguous when time-dependent driving is present. In Ref. 11, they argue that in nonsteady states, the tunneling region has some energy ("energy reactance") and it is unclear whether to ascribe that to the "system" or the "bath." This, they argue, leads to an ambiguity in the definition of the heat, which they propose is fixed by ascribing half the energy of the tunneling region to the bath. Although this definition of heat does agree with the laws of thermodynamics, it is not clear whether their prescription is applicable to models other than the one they consider. Reference 12 proposes to experimentally measure this energy reactance in order to determine empirically what partitioning leads to the correct heat current definition.

In Ref. 13, Nitzan and collaborators consider a driven resonant level model and show that the problem of separately defining "system" and "bath" in the strong-coupling regime is circumvented by considering as the system everything that is influenced by the externally driven energy level and rebut the claims of a violation of the heat theorem put forward in Ref. 10. Their book-keeping ${ }^{13}$ is similar to that originally put forward in the equilibrium case by Friedel. ${ }^{14}$ Finally, Ref. 15 uses a scattering approach to similarly circumvent the problem of system/bath definitions for adiabatically driven open quantum systems, expressing changes in the entropy of the system in terms of asymptotic observables at infinity.

In this article, we investigate the applicability of Nernst's heat theorem (A) to a general class of open quantum systems, consisting of a finite system of independent fermions with arbitrary Hamiltonian coupled to one or more equilibrium fermion reservoirs (which need not be in equilibrium with each other). Both the equilibrium case and the case of a nonequilibrium steady state are considered. We consider a general partitioning of the entire system into subsystem and reservoir(s), where the subsystem can be any finite subspace of the total Hilbert space. We show that no ambiguity arises from the partitioning either in equilibrium or in a nonequilibrium steady state, and give proofs of the heat theorem for both cases.

\section{THEORETICAL METHODS}

We consider a generic open quantum system of independent fermions coupled to one or more macroscopic fermion reservoirs. The reservoirs are separately in thermodynamic equilibrium but need not be in equilibrium with each other.

\section{A. Hamiltonian}

The Hamiltonian is

$$
\hat{H}=\hat{H}_{\text {sys }}+\hat{H}_{\text {res }}+\hat{H}_{\text {s-r }} \text {, }
$$

where $^{16}$

$$
\hat{H}_{\text {sys }}=\sum_{i, j \in \text { sys }}\left(H_{\text {sys }}\right)_{i j} d_{i}^{\dagger} d_{j}
$$

is a generic 1-body Hamiltonian for a finite spatial domain, with $\left(H_{\text {sys }}\right)_{i j}^{*}=\left(H_{\text {sys }}\right)_{j i}$,

$$
\hat{H}_{\text {res }}=\sum_{\alpha=1}^{M} \sum_{k \in \alpha} \epsilon_{k} d_{k}^{\dagger} d_{k}
$$

is the Hamiltonian describing $M$ fermion reservoirs, and

$$
\hat{H}_{\mathrm{s}-\mathrm{r}}=\sum_{i \in \text { sys }} \sum_{\alpha=1}^{M} \sum_{k \in \alpha}\left(V_{i k} d_{i}^{\dagger} d_{k}+\text { H.c. }\right)
$$

describes the system-reservoir coupling. Here, $d_{i}$ and $d_{j}^{\dagger}$ are fermion annihilation and creation operators obeying canonical anticommutation relations. For simplicity and mathematical rigor, we assume that the Hilbert space on which $H_{\text {sys }}$ acts is finite.

We note that the partitioning of the total system into "system" and "reservoir(s)" is to some extent arbitrary, except that the system is finite and the reservoirs are typically taken to be infinite. The division between system and reservoirs should be understood as a division of the Hilbert space, not as a division of the Hamiltonian. The open quantum system, coupled to its reservoir(s), is no longer described by a Hermitian Hamiltonian but instead is described in terms of Green's functions.

\section{B. Green's functions}

The dynamics of the open quantum system (1) are described by the retarded Green's function, ${ }^{17,18}$

$$
G_{i j}(t)=-i \theta(t)\left\langle\left\{d_{i}(t), d_{j}^{\dagger}(0)\right\}\right\rangle,
$$

where $\langle\cdots\rangle$ denotes the quantum and statistical average, and we work in units where $\hbar=1$. Although the matrix elements of the Green's function are defined for any pair of annihilation and creation operators in the Hilbert space of Eq. (1), only the finite submatrix acting on the system Hilbert space is needed to compute quantities of interest in the open quantum system, and $G$ shall denote that submatrix for the remainder of this article. For additional information on Green's function theory, see Appendix A.

In this article, we consider systems in equilibrium or in a nonequilibrium steady state, so it is useful to work with the Fourier transform $G(\omega)$, given by ${ }^{17,18}$

$$
G(\omega)=\left[\mathbb{1} \omega-H_{\text {sys }}-\Sigma(\omega)\right]^{-1},
$$

where the retarded self-energy

$$
\Sigma(\omega) \equiv \sigma(\omega)-i \Gamma(\omega) / 2-i \eta \mathbb{1}
$$

accounts for the system-reservoir coupling, with $\sigma(\omega)=\sigma^{\dagger}(\omega)$ and $\Gamma(\omega)=\Gamma^{\dagger}(\omega)$. Here, $\eta=0^{+}$is a positive infinitesimal and $\mathbb{1}$ is the unit operator. The broadening of the states of the system is determined by

$$
\Gamma(\omega)=\sum_{\alpha=1}^{M} \Gamma^{\alpha}(\omega)
$$

where

$$
\Gamma_{i j}^{\alpha}(\omega)=2 \pi \sum_{k \in \alpha} V_{i k} V_{j k}^{*} \delta\left(\omega-\epsilon_{k}\right)=\left.2 \pi \overline{V_{i k} V_{j k}^{*}}\right|_{k \in \alpha} \rho_{\alpha}(\omega)
$$

is the partial width function due to coupling with reservoir $\alpha$, where $\rho_{\alpha}(\omega)$ is the density of states of reservoir $\alpha$. The rhs of Eq. (9) is an 
exact result of Green's function theory, not an approximation, but agrees with Fermi's golden rule. The real part of the shifts of the system energy levels due to coupling with the reservoirs is determined by

$$
\sigma_{i j}(\omega)=\sum_{\alpha=1}^{M} \sum_{k \in \alpha} \mathcal{P} \frac{V_{i k} V_{j k}^{*}}{\omega-\epsilon_{k}},
$$

where $\mathcal{P}$ denotes the principal part. In the thermodynamic limit for the reservoirs, the self-energy $\Sigma(\omega)$ becomes a smooth function of $\omega$ as the sums over poles in Eqs. (9) and (10) may be replaced by integrals. All quantities calculated from the Green's functions are well defined in this limit.

\section{Energy spectrum}

The spectral function of the system is defined as

$$
A(\omega) \equiv \delta(\omega-H),
$$

whose trace gives the energy spectrum. A fundamental result of Green's function theory (see Appendix A) is

$$
A(\omega)=\frac{i}{2 \pi}\left[G(\omega)-G^{\dagger}(\omega)\right]
$$

which may be decomposed as

$$
A(\omega)=\sum_{\alpha=1}^{M} A_{\alpha}(\omega)+\sum_{\ell}|\ell\rangle\langle\ell| \delta\left(\omega-\omega_{\ell}\right),
$$

where

$$
A_{\alpha}(\omega)=\frac{1}{2 \pi} G(\omega) \Gamma^{\alpha}(\omega) G^{\dagger}(\omega)
$$

is the partial spectral function due to scattering states incident on the system from reservoir $\alpha$, and the sum over $\ell$ includes any localized states that are not broadened due to the coupling with the reservoir(s). The localized states, if any, satisfy

$$
\left[\omega_{\ell}-H_{\text {sys }}-\sigma\left(\omega_{\ell}\right)+i \Gamma\left(\omega_{\ell}\right) / 2\right]|\ell\rangle=0,
$$

and their energies are denoted by $\omega_{\ell}$. A similar condition was recently analyzed in the context of destructive quantum interferences. ${ }^{19,2}$

The density of states of the open quantum system is the trace over the system Hilbert space of the spectral function,

$$
g(\omega)=\operatorname{Tr}_{\text {sys }}\{A(\omega)\}=g_{\text {reg }}(\omega)+\sum_{\ell} \delta\left(\omega-\omega_{\ell}\right),
$$

where

$$
g_{\text {reg }}(\omega)=\sum_{\alpha=1}^{M} g_{\alpha}(\omega)
$$

is the nonsingular part of the spectrum, and $\mathrm{d}^{21-23}$

$$
g_{\alpha}(\omega)=\operatorname{Tr}_{\text {sys }}\left\{A_{\alpha}(\omega)\right\}
$$

is the partial density of states of the system due to scattering states incident on the system from reservoir $\alpha$. It should be emphasized that an open quantum system is a subsystem of a larger system, and $g(\omega)$ gives the energy spectrum of the whole system projected onto the Hilbert space of the subsystem. Other prescriptions for partitioning into subsystem and environment are also possible.

Similarly, the local density of states is given by

$$
g(\omega ; \mathbf{x})=\langle\mathbf{x}|A(\omega)| \mathbf{x}\rangle=\sum_{\alpha=1}^{M} g_{\alpha}(\omega ; \mathbf{x})+\sum_{\ell}\left|\psi_{\ell}(\mathbf{x})\right|^{2} \delta\left(\omega-\omega_{\ell}\right),
$$

where the local partial density of states associated with reservoir $\alpha$ is $^{21-23}$

$$
g_{\alpha}(\omega ; \mathbf{x})=\left\langle\mathbf{x}\left|A_{\alpha}(\omega)\right| \mathbf{x}\right\rangle .
$$

\section{Thermodynamics}

In the present article, open quantum systems refer to systems which may exchange both particles and energy with the reservoir(s). The statistical ensemble used to describe such thermodynamic systems is the grand canonical ensemble. The grand canonical potential $\Omega=E-T S-\mu N$ is the characteristic state function ${ }^{24}$ for such an ensemble from which all thermodynamic averages may be calculated. The grand canonical potential of an open quantum system in thermodynamic equilibrium at absolute temperature $T$ and chemical potential $\mu$ is given by

$$
\Omega(\mu, T)=-k_{B} T \int d \omega g(\omega) \ln \left[1+e^{-\beta(\omega-\mu)}\right],
$$

where $k_{B}$ is Boltzmann's constant and $\beta=1 / k_{B} T$. The entropy of the system is given by

$$
\begin{aligned}
S=-\left.\frac{\partial \Omega}{\partial T}\right|_{\mu} & =\int d \omega g(\omega) s(f(\omega)) \\
& =\int d \omega g_{\text {reg }}(\omega) s(f(\omega))+\sum_{\ell} s\left(f\left(\omega_{\ell}\right)\right),
\end{aligned}
$$

where

$$
s(f)=-k_{B}[f \ln f+(1-f) \ln (1-f)]
$$

and

$$
f(\omega)=\left[e^{\beta(\omega-\mu)}+1\right]^{-1}
$$

is the equilibrium Fermi-Dirac distribution of the reservoir(s). Similarly, one can define the local entropy density ${ }^{26}$

$$
\begin{aligned}
S(\mathbf{x}) & =\int d \omega g(\omega ; \mathbf{x}) s(f(\omega)) \\
& =\sum_{\alpha=1}^{M} \int d \omega g_{\alpha}(\omega ; \mathbf{x}) s(f(\omega))+\sum_{\ell}\left|\psi_{\ell}(\mathbf{x})\right|^{2} s\left(f\left(\omega_{\ell}\right)\right),
\end{aligned}
$$

which satisfies $S=\int_{\text {sys }} d^{3} \times S(\mathbf{x})$.

The definition of local entropy and entropy of a subsystem ${ }^{27}$ given here, wherein entropy is additive over subsystems, is consistent with the ordinary thermodynamic definition of entropy embodied in the first line of Eq. (22) but differs from the von Neumann entropy computed from the reduced density matrix of a subsystem. 
In the later definition, entanglement of the subsystem with the rest of the system leads to entanglement entropy that is not additive over subsystems.

\section{E. Nonequilibrium steady states}

The equilibrium entropy formulas (22) and (25) can be generalized to the case of an open quantum system in a nonequilibrium steady state. ${ }^{28,29}$ Succinctly, the nonequilibrium steady state of a quantum scattering problem for independent quantum particles can be decomposed into independent contributions from the scattering states incident from each reservoir, which coexist in real space, but are orthogonal in Hilbert space. The nonequilibrium entropy of the open quantum system is ${ }^{28,2}$

$$
S=\sum_{\alpha=1}^{M} \int d \omega g_{\alpha}(\omega) s\left(f_{\alpha}(\omega)\right)+\sum_{\ell} s\left(f_{\ell}\right)
$$

where $f_{\alpha}(\omega)=\left[e^{\beta_{\alpha}\left(\omega-\mu_{\alpha}\right)}+1\right]^{-1}$ is the Fermi-Dirac distribution of reservoir $\alpha$ and $f_{\ell}$ is the occupancy of the $\ell$ th localized state. The local nonequilibrium entropy density is ${ }^{28,29}$

$$
S(\mathbf{x})=\sum_{\alpha=1}^{M} \int d \omega g_{\alpha}(\omega ; \mathbf{x}) s\left(f_{\alpha}(\omega)\right)+\sum_{\ell}\left|\psi_{\ell}(\mathbf{x})\right|^{2} s\left(f_{\ell}\right),
$$

which satisfies $S=\int_{\text {sys }} d^{3} x S(\mathbf{x})$, with $S$ being the nonequilibrium entropy given by Eq. (26).

\section{THIRD LAW FOR EQUILIBRIUM SYSTEMS}

Theorem 1 (Third law of thermodynamics). For an open quantum system with a finite-dimensional Hilbert space,

$$
\lim _{T \rightarrow 0} S(\mu, T)=0
$$

almost everywhere for $\mu \in \mathcal{R}$.

Proof. We consider the first term on the rhs of Eq. (22),

$$
\lim _{T \rightarrow 0} S_{\text {reg }}(\mu, T)=\lim _{T \rightarrow 0} \int_{-\infty}^{\infty} d \omega g_{\text {reg }}(\omega) s(f(\omega))
$$

and note that

$$
\int_{-\infty}^{\infty} d \omega g_{\text {reg }}(\omega)=N_{\text {reg }} \leq N_{\mathcal{H}}
$$

where $N_{\mathcal{H}}=\operatorname{dim}\{\mathcal{H}\}$ is the dimension of the Hilbert space $\mathcal{H}$ of the system. The Fermi function $\lim _{T \rightarrow 0} f(\omega) \rightarrow 1-\Theta(\omega-\mu)$, where $\Theta$ is the Heaviside step function. Therefore,

$$
\begin{aligned}
\lim _{T \rightarrow 0} S_{\text {reg }}(\mu, T) & =\lim _{f \rightarrow 1} \int_{-\infty}^{\mu} d \omega g_{\text {reg }}(\omega) s(f)+\lim _{f \rightarrow 0} \int_{\mu}^{\infty} d \omega g_{\text {reg }}(\omega) s(f) \\
& =0
\end{aligned}
$$

since $\lim _{f \rightarrow 1} s(f)=\lim _{f \rightarrow 0} s(f)=0$ and the integral of $g(\omega)$ is bounded by the dimension of the Hilbert space [Eq. (30)]. A similar result, restricted to the resonant level model, was derived in Ref. 13.
The second term from Eq. (22) has the entropy contribution from the localized states which vanish as $T \rightarrow 0$ when $\mu \neq \omega_{\ell}$ since

$$
\lim _{f \rightarrow 0} s\left(f\left(\omega_{\ell}\right)\right)=\lim _{f \rightarrow 1} s\left(f\left(\omega_{\ell}\right)\right)=0,
$$

and when $\mu=\omega_{\ell}$, we get

$$
S_{\mathrm{loc}}=\lim _{T \rightarrow 0} s\left(f\left(\mu=\omega_{\ell}\right)\right)=k_{B} \log (2),
$$

and if there are multiple localized states at $\omega=\omega_{\ell}$, we denote the degeneracy as $g_{\ell}$ and may write

$$
S_{\mathrm{loc}}=g_{\ell} k_{B} \log (2) \text {. }
$$

The points $\mu=\omega_{\ell}$ have zero measure for $\mu \in \mathcal{R}$, and this completes the proof.

Theorem 1 constitutes the general form of the third law of thermodynamics for open quantum systems and is the central result of this paper. Figure 1 illustrates the behavior of $S(\mu, T)$ as $T \rightarrow 0$ for a model open quantum system consisting of a benzene ring coupled to an electron reservoir.

\section{A. Fully open quantum systems}

A strong form of the third law of thermodynamics can be shown to hold for fully open quantum systems.

Definition 1. A fully open quantum system is any system for which Eq. (15) has no solutions for $\omega_{\ell} \in \mathcal{R}$.

In a fully open quantum system, all of the energy eigenstates of the system are broadened due to coupling to the reservoir; there are no localized states.

Corollary 1. For a fully open quantum system with a finitedimensional Hilbert space,

$$
\lim _{T \rightarrow 0} S(\mu, T)=0 \forall \mu .
$$

Proof. For a fully open system, Eq. (30) holds with $N_{\text {reg }}=N_{\mathcal{H}}$ and there are no localized states. Proof follows directly from Eq. (31) and Theorem 1 holds $\forall \mu \in \mathcal{R}$.

Since $S$ is a smooth function of $\mu \in \mathcal{R}$ and $T \in(0, \infty)$, we provide the leading-order low-temperature expansion, ${ }^{30}$

$$
S(\mu, T)=\frac{\pi^{2}}{3} g(\mu) k_{B}^{2} T .
$$

$S$ scales as a higher power of $T$ as $T \rightarrow 0$ if $g(\mu)=0$.

\section{Sufficient condition for a fully open quantum system}

A sufficient condition for a fully open quantum system is

$$
\Gamma(\omega)|\psi\rangle \neq 0 \quad \forall|\psi\rangle \in \mathcal{H},
$$




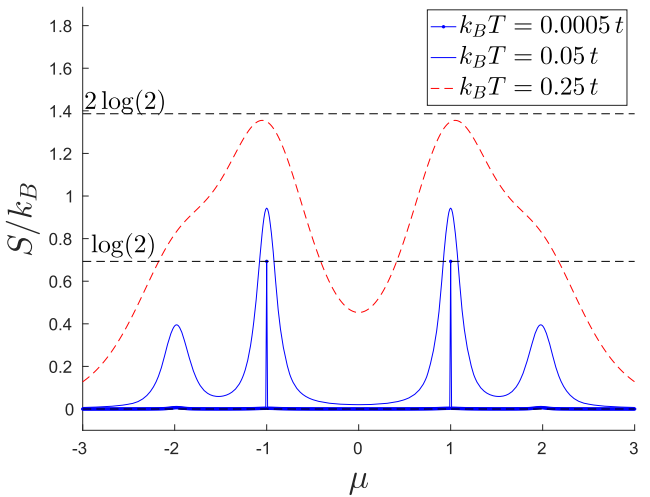

(a)

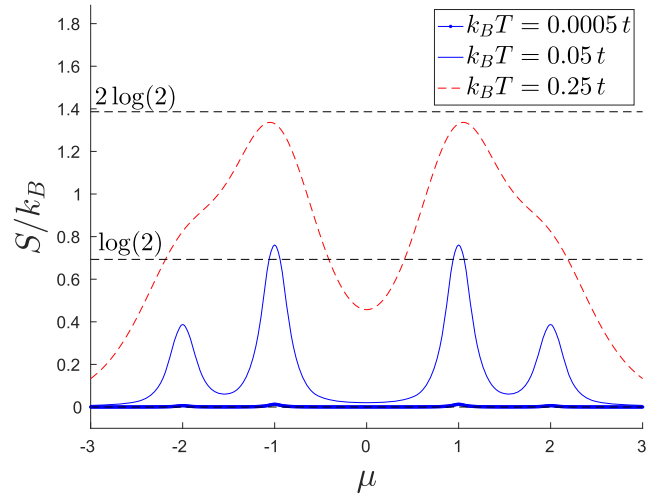

(b)

FIG. 1. Entropy $S$ of an open quantum system consisting of a benzene ring coupled to an equilibrium electron reservoir, plotted as a function of the chemical potential $\mu$ of the reservoir for several temperatures. The molecule is modeled using Hückel theory (tight-binding approximation, see Appendix B), and energies are expressed in units of the nearest-neighbor hybridization $t$. (a) Generic open system with $\Gamma_{11}=t$ and all other matrix elements of $\Gamma$ zero, illustrating the effect of the localized states at $\mu / t= \pm 1$. (b) Fully open quantum system with $\Gamma=(t / 6) \mathbb{1}$, illustrating the strong form of the third law, $S(T) \rightarrow 0$ as $T \rightarrow 0 \forall \mu$.

where $\mathcal{H}$ denotes the Hilbert space of $H_{\text {sys. }}$ Equation (37) holds if $\operatorname{rank}\{\Gamma\}=\operatorname{dim} \mathcal{H}$ since $\Gamma \geq 0 .{ }^{31}$ Figure $1(\mathrm{~b})$ illustrates the case of a fully open quantum system, for the same model of a benzene ring coupled to a reservoir, but with $\Gamma=\gamma \mathbb{1}$, so that $\operatorname{rank}\{\Gamma\}=6$. Note the absence of any localized states in the entropy spectrum.

\section{Example}

Equation (37) is a sufficient condition for a fully open quantum system but is not a necessary condition. To see that this is the case, consider the following example. Let the system-reservoir coupling be in the broad-band limit so that $\sigma(\omega)=0$ and $\Gamma(\omega)=$ const. Let $|v\rangle$ be an eigenstate of $H_{\text {sys }}, H_{\text {sys }}|v\rangle=\varepsilon_{v}|v\rangle$, and suppose

$$
\langle v|\Gamma| v\rangle \neq 0 \quad \forall v .
$$

Then, it is straightforward to show that there exist no solutions to Eq. (15) for real energies, provided the eigenenergies $\varepsilon_{v}$ of $H_{\text {sys }}$ are nondegenerate. The following rank-1 $\Gamma$ matrix is an example leading to a fully open quantum system with a nondegenerate spectrum:

$$
\Gamma=\gamma|\gamma\rangle\langle\gamma|, \text { where }|\gamma\rangle=\sum_{v} C_{v}|v\rangle,
$$

and $C_{v} \neq 0$ are complex numbers.

However, if the energy eigenvalue $\varepsilon_{v}$ is degenerate and the support of $\Gamma$ does not span the degenerate subspace, then there will be at least one localized state of energy $\varepsilon_{v}$. This case is illustrated in Fig. 1(a).

\section{THIRD LAW FOR NONEQUILIBRIUM SYSTEMS}

For nonequilibrium systems, one also needs the "lesser" Green's function ${ }^{17,26}$

$$
G_{i j}^{<}(t)=i\left\langle d_{j}^{\dagger}(0) d_{i}(t)\right\rangle
$$

which determines the occupancies of the states. Its Fourier transform obeys the Keldysh equation,

$$
G^{<}(\omega)=G(\omega) \Sigma^{<}(\omega) G^{\dagger}(\omega)
$$

where the lesser self-energy is given by

$$
\Sigma^{<}(\omega)=i \sum_{\alpha=1}^{M} \Gamma^{\alpha}(\omega) f_{\alpha}(\omega)+2 i \eta \sum_{\ell}|\ell\rangle\langle\ell| f_{\ell},
$$

where $f_{\alpha}(\omega)$ and $f_{\ell}$ are the Fermi-Dirac distribution of reservoir $\alpha$ and the nonequilibrium occupancy of the $\ell$ th localized state, respectively, as discussed in Sec. II E. Inserting Eq. (42) in Eq. (41) and using Eq. (14), one finds

$$
G^{<}(\omega)=2 \pi i\left[\sum_{\alpha=1}^{M} A_{\alpha}(\omega) f_{\alpha}(\omega)+\sum_{\ell} f_{\ell}|\ell\rangle\langle\ell| \delta\left(\omega-\omega_{\ell}\right)\right] .
$$

The particle density $N(\mathbf{x})$ and energy density $E(\mathbf{x})$ of the nonequilibrium quantum system are given by ${ }^{26}$

$$
N(\mathbf{x})=\int d \omega g(\omega ; \mathbf{x}) f(\omega, \mathbf{x})
$$

$$
E(\mathbf{x})=\int d \omega g(\omega ; \mathbf{x}) \omega f(\omega, \mathbf{x})
$$

where

$$
f(\omega ; \mathbf{x}) \equiv \frac{\left\langle\mathbf{x}\left|G^{<}(\omega)\right| \mathbf{x}\right\rangle}{2 \pi i g(\omega ; \mathbf{x})}= \begin{cases}f_{\ell}, & \omega=\omega_{\ell} \\ \frac{1}{g(\omega ; \mathbf{x})} \sum_{\alpha} g_{\alpha}(\omega ; \mathbf{x}) f_{\alpha}(\omega), & \omega \neq \omega_{\ell}\end{cases}
$$

is the local nonequilibrium distribution function of the system. ${ }^{26,32}$ Note that $f(\omega ; \mathbf{x})$ may be discontinuous at $\omega=\omega_{\ell}$. Equation (44) 
holds quite generally, while Eq. (45) holds for the case of independent fermions.

\section{A. Local thermodynamic variables $T(\mathbf{x}), \mu(\mathbf{x})$}

In order to address the applicability of the 3rd law of thermodynamics in systems out of equilibrium, one needs a concept of local temperature. ${ }^{23,26,30,31,33-37}$ Here, we define the local temperature $T(\mathbf{x})$ and chemical potential $\mu(\mathbf{x})$ as those measured by a floating broadband thermoelectric probe coupled weakly to the system at the point $\mathbf{x}$ (see Refs. 23, 26, and 31 for discussion). The floating probe consists of an equilibrium electron reservoir whose temperature and chemical potential are adjusted such that the net flow of charge and heat into the probe is zero. Under these conditions, the FermiDirac distribution of the probe reservoir $f_{p}(\omega ; \mathbf{x})$ defines $T(\mathbf{x})$ and $\mu(\mathbf{x})$ via

$$
f_{p}(\omega ; \mathbf{x})=\left(e^{\beta(\mathbf{x})[\omega-\mu(\mathbf{x})]}+1\right)^{-1} .
$$

$f_{p}(\omega ; \mathbf{x})$ is the unique equilibrium distribution that reproduces the particle and energy densities of the nonequilibrium system given by Eqs. (44) and (45), respectively. ${ }^{23,26}$ For a system of independent fermions, it was shown ${ }^{31}$ that the probe distribution function $f_{p}(\omega ; \mathbf{x})$ always exists and is unique; for interacting fermions, the uniqueness and existence of $f_{p}(\omega ; \mathbf{x})$ was proven for the case of weak probe-system coupling only.

Definition 2. Let $S_{p}(\mathbf{x})$ be the entropy density of the fictitious local equilibrium state obtained by inserting the probe distribution function $f_{p}(\omega ; \mathbf{x})$, Eq. (47), into the equilibrium formula, Eq. (25).

$S_{p}(\mathbf{x})$ so defined clearly satisfies Theorem 1.

Definition 3. Let $S_{s}(\mathbf{x})$ be a measure of the local nonequilibrium entropy density of the system defined by inserting the local nonequilibrium distribution function $f(\omega ; \mathbf{x})$, Eq. (46), directly into the equilibrium formula, Eq. (25).

$S_{s}(\mathbf{x})$ so defined was introduced previously in Ref. 26 and may be thought of as the local entropy density inferred by an observer with strictly local knowledge of the nonequilibrium state of the system. ${ }^{28,29}$

\section{Maximum entropy principle: $S_{s}(x) \leq S_{p}(x)$}

The fictitious local equilibrium distribution $f_{p}(\omega ; \mathbf{x})$ satisfies the same constraints [Eqs. (44) and (45)] as the actual nonequilibrium distribution $f(\omega ; \mathbf{x})$. Therefore, by the maximum entropy principle, $S_{p}(\mathbf{x}) \geq S_{s}(\mathbf{x})$. For a detailed variational argument for the case of a fully open quantum system, see Ref. 26.

Theorem 2 (Third law for nonequilibrium systems). For an open quantum system in a nonequilibrium steady state, and with a finite-dimensional Hilbert space,

$$
S(\mathbf{x}) \rightarrow 0 \text { as } T(\mathbf{x}) \rightarrow 0,
$$

almost everywhere in space.
Proof. $S(\mathbf{x})$ is defined by Eq. (27). The contributions of any localized states to $S(\mathbf{x})$ and $S_{s}(\mathbf{x})$ are identical. Therefore, any difference between $S(\mathbf{x})$ and $S_{s}(\mathbf{x})$ is due to scattering states. From the concavity of the function $s(f)$ defined by Eq. (23), it follows that $S_{s}(\mathbf{x}) \geq S(\mathbf{x})$ because

$$
s(f) \geq \sum_{\alpha=1}^{M} \lambda_{\alpha} s\left(f_{\alpha}\right)
$$

where

$$
f(\omega ; \mathbf{x})=\sum_{\alpha=1}^{M} \lambda_{\alpha}(\omega ; \mathbf{x}) f_{\alpha}(\omega), \quad \sum_{\alpha=1}^{M} \lambda_{\alpha}=1,
$$

and $\lambda_{\alpha}(\omega ; \mathbf{x})=g_{\alpha}(\omega ; \mathbf{x}) / g(\omega ; \mathbf{x}) \geq 0$. However, $S_{s}(\mathbf{x}) \leq S_{p}(\mathbf{x})$ by the maximum entropy principle. Therefore, $S(\mathbf{x}) \leq S_{p}(\mathbf{x})$.

Finally, the limiting behavior of $S_{p}(\mathbf{x})$ can be obtained from the Sommerfeld expansion of Eq. (25),

$$
S_{p}(\mathbf{x}) \stackrel{T(\mathbf{x}) \rightarrow 0}{\sim} \frac{\pi^{2}}{3} k_{B}^{2} T(\mathbf{x}) \sum_{\alpha=1}^{M} g_{\alpha}(\mu(\mathbf{x}) ; \mathbf{x})+\sum_{\ell}\left|\psi_{\ell}(\mathbf{x})\right|^{2} s\left(f_{p}\left(\omega_{\ell} ; \mathbf{x}\right)\right),
$$

provided $\sum_{\alpha} g_{\alpha}(\mu(\mathbf{x}) ; \mathbf{x}) \neq 0$, and if it is zero, the first term on the rhs vanishes as a higher power of $T(\mathbf{x})$. Therefore,

$$
\lim _{T(\mathbf{x}) \rightarrow 0} S(\mathbf{x})= \begin{cases}0, & \mu(\mathbf{x}) \neq \omega_{\ell}, \\ k_{B} \ln 2 \sum_{\ell}\left|\psi_{\ell}(\mathbf{x})\right|^{2}, & \mu(\mathbf{x})=\omega_{\ell} .\end{cases}
$$

In a nonequilibrium system, where $\mu(\mathbf{x})$ varies as a continuous function of position, ${ }^{38,39}$ the contribution from localized states in Eq. (52) vanishes everywhere in space except on a set of measure zero.

\section{CONCLUSIONS}

The third law of thermodynamics (Nernst's heat theorem) is shown to hold true for open quantum systems, both in and out of equilibrium. This is proven for a generic open quantum system of independent fermions with strong coupling to reservoirs but no time-dependent external driving.

Our analysis of the third law was shown to hold for quite general partitioning of Hilbert space into an open subsystem and an environment [reservoir(s)]. It is important to emphasize that such a partitioning is well defined in Hilbert space, but that physical observables relevant for thermodynamics may involve both system and reservoir degrees of freedom.

The laws of thermodynamics are arguably the most fundamental of all physical principles, more general than any particular theory, such as quantum mechanics or general relativity. For this reason, claims of violations of the laws of thermodynamics in open and/or nonequilibrium quantum systems should be treated with appropriate skepticism, and subjected to the most stringent examination before they are taken at face value.

\section{ACKNOWLEDGMENTS}

This work was supported by the U.S. Department of Energy (DOE), Office of Science under Award No. DE-SC0006699. 


\section{APPENDIX A: GREEN'S FUNCTION THEORY}

In this appendix, some background information on nonequilibrium Green's functions (NEGF) is provided, and the relation between the spectral function $A(\omega)$ and the retarded Green's function $G(\omega)$ is derived. In NEGF theory, the statistical and quantum average in Eq. (5) is computed using the $S$-matrix, evaluated on the Schwinger-Keldysh time contour (see the textbooks ${ }^{17,40,41}$ for details).

The starting point for the derivation of Eq. (12) connecting the spectral function and the anti-Hermitian part of the retarded Green's function is the equation of motion for $G$,

$$
i \frac{\partial}{\partial t} G_{n m}(t)=\delta(t) \delta_{n m}+i \theta(t)\left\langle\left\{\left[\hat{H}, d_{n}(t)\right], d_{m}^{\dagger}(0)\right\}\right\rangle,
$$

where $\hat{H}$ is the full Hamiltonian given by Eq. (1), which we write as

$$
\hat{H}=\sum_{j, \ell} H_{j \ell} d_{j}^{\dagger} d_{\ell} .
$$

The commutator in Eq. (A1) is straightforward to evaluate

$$
\left[\hat{H}, d_{n}\right]=-\sum_{\ell} H_{n \ell} d_{\ell}
$$

Inserting Eq. (A3) into Eq. (A1), one finds

$$
i \frac{\partial}{\partial t} G_{n m}(t)=\delta(t) \delta_{n m}-\sum_{\ell} H_{n \ell} G_{\ell m}(t) .
$$

Taking the Fourier transform of Eq. (A4) and solving for G, one obtains the result

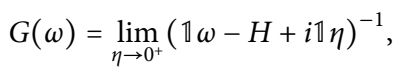

where the infinitesimal term $\eta$ is needed to generate the step function $\theta(t)$ upon Fourier transforming back to the time domain.

Let the eigenstates and eigenvalues of $H$ be written as $H|v\rangle=\varepsilon_{v}|v\rangle$. Using the completeness relation for the energy eigenstates in Eq. (A5), one obtains the spectral representation of $G$,

$$
G(\omega)=\lim _{\eta \rightarrow 0^{+}} \sum_{v} \frac{|v\rangle\langle v|}{\omega-\varepsilon_{v}+i \eta} .
$$

Finally, one finds

$$
\begin{aligned}
\frac{i}{2 \pi}\left[G(\omega)-G^{\dagger}(\omega)\right] & =\lim _{\eta \rightarrow 0^{+}} \frac{\eta}{\pi} \sum_{v} \frac{|v\rangle\langle v|}{\left(\omega-\varepsilon_{v}\right)^{2}+\eta^{2}} \\
& =\sum_{v}|v\rangle\langle v| \delta\left(\omega-\varepsilon_{v}\right) \equiv \delta(\omega-H),
\end{aligned}
$$

which completes the derivation. For an analogous derivation of Eq. (6) for the Green's function submatrix using the equations of motion method, see Ref. 18 or the textbooks, Refs. 17, 40, and 41.

\section{APPENDIX B: BENZENE MOLECULAR JUNCTION}

As a specific example of an open quantum system, a molecular junction consisting of a single benzene molecule coupled to a macroscopic electron reservoir is analyzed in Fig. 1. The molecule is modeled using Hückel theory, with Hamiltonian

$$
H_{\text {sys }}=-\sum_{j=1}^{6}\left(t e^{i \pi \Phi / 3 \phi_{0}} d_{j}^{\dagger} d_{j+1}+\text { H.c. }\right) \text {, }
$$

where the benzene ring is threaded by a magnetic flux $\Phi$ and $\phi_{0}=h c / e$ is the magnetic flux quantum. The system is depicted in Fig. 2. For benzene, the nearest-neighbor coupling ${ }^{42}$ is taken as $t=2.7 \mathrm{eV}$ and the fermion annihilation operators satisfy periodic boundary conditions, $d_{0}=d_{6}, d_{7}=d_{1}$. For simplicity, spin is neglected, which reduces the total entropy of the system by a factor of two.

\section{Fully open quantum system}

Let us first consider the case of a fully open quantum system with retarded self-energy

$$
\Sigma_{n m}(\omega)=-\frac{i \gamma}{2} \delta_{n m}
$$

For this case, the density of states may be calculated in the closed form as

$$
g(\omega, \Phi)=\frac{6}{\pi} \frac{K(\omega) Q(\omega)-L(\omega) P(\omega, \Phi)}{P(\omega, \Phi)^{2}+Q(\omega)^{2}},
$$

where the four functions $K, L, P$, and $Q$ are given by

$$
\begin{aligned}
& K(\omega)=x\left(x^{2}-3 y^{2}-t^{2}\right)\left(x^{2}-y^{2}-3 t^{2}\right)-2 x y^{2}\left(3 x^{2}-y^{2}-t^{2}\right), \\
& L(\omega)=2 x^{2} y\left(x^{2}-3 y^{2}-t^{2}\right)+y\left(3 x^{2}-y^{2}-t^{2}\right)\left(x^{2}-y^{2}-3 t^{2}\right), \\
& P(\omega, \Phi)=x^{2}\left(x^{2}-3 y^{2}-3 t^{2}\right)^{2}-y^{2}\left(3 x^{2}-y^{2}-3 t^{2}\right)^{2}-4 t^{6} \cos ^{2}(\varphi),
\end{aligned}
$$

and

$$
Q(\omega)=2 x y\left(x^{2}-3 y^{2}-3 t^{2}\right)\left(3 x^{2}-y^{2}-3 t^{2}\right),
$$

respectively, where $x \equiv \omega, y \equiv \gamma / 2$, and $\varphi \equiv \pi \Phi / \phi_{0}$. It can be shown that $P(\omega, \Phi)^{2}+Q(\omega)^{2} \neq 0 \forall \omega \in \mathcal{R}$, so $g(\omega, \Phi)$ is welldefined everywhere along the real- $\omega$ axis. This case is illustrated in Fig. 1(b).

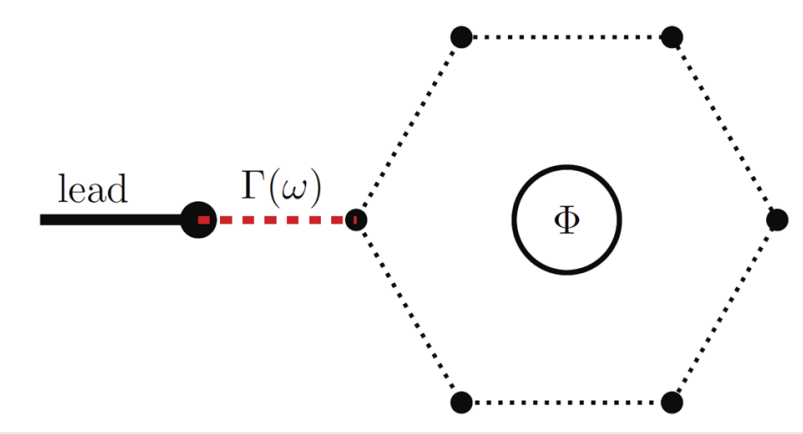

FIG. 2. An open quantum system consisting of a benzene molecule threaded by a magnetic flux $\Phi$, coupled to a single macroscopic reservoir (lead). 


\section{Open system with localized states}

Next, let us consider coupling the benzene molecule to the reservoir via a single covalent bond, as shown in Fig. 2. The retarded self-energy for this case is

$$
\Sigma_{n m}(\omega)=-\frac{i \gamma}{2} \delta_{m 1} \delta_{n 1}
$$

Since $\operatorname{rank}\{\Gamma\}=1$, localized states occur whenever there is a degeneracy in the spectrum of $H_{\text {sys }}$, as discussed in Sec. III A 2. The density of states is given by

$$
g(\omega, \Phi)=-\frac{1}{\pi t} \frac{-U E^{2}\left(E^{2}-3\right)^{2}\left(E^{4}+3\right)-4 U\left(5 E^{4}-12 E^{2}+3\right) \cos ^{2}(\varphi)}{\left[4 \cos ^{2}(\varphi)-E^{2}\left(E^{2}-3\right)^{2}\right]^{2}+\left[U E\left(E^{2}-3\right)\left(E^{2}-1\right)\right]^{2}},
$$

where $E \equiv \omega / t, U \equiv \gamma / 2 t$, and again $\varphi \equiv \pi \Phi / \phi_{0}$. A careful analysis of the denominator in Eq. (B9) reveals that $g(\omega, \Phi)$ is singular for $\left(E, \Phi / \phi_{0}\right)=( \pm 1, n),\left(E, \Phi / \phi_{0}\right)=( \pm \sqrt{3}, n+1 / 2)$, and $\left(E, \Phi / \phi_{0}\right)$ $=(0, n+1 / 2)$, where $n$ is an integer. We treat the two special values of the flux separately in Appendixes B $2 \mathrm{a}$ and B 2 b.

\section{a. Integer flux: $\Phi=\boldsymbol{n} \phi_{0}$}

For $\Phi=n \phi_{0}, g(E)$ is regular except at $E= \pm 1$. Evaluating the limiting behavior as $\Phi \rightarrow n \phi_{0}$, one finds

$$
\begin{aligned}
g\left(\omega, \Phi=n \phi_{0}\right)= & \frac{\delta(E-1)}{t}+\frac{\delta(E+1)}{t} \\
& +\frac{U}{\pi t} \frac{E^{6}-4 E^{4}+3 E^{2}+12}{\left(E^{2}-1\right)^{2}\left(E^{2}-4\right)^{2}+U^{2} E^{2}\left(E^{2}-3\right)^{2}} .
\end{aligned}
$$

For this case,

$$
\lim _{T \rightarrow 0} S(\mu, T)= \begin{cases}k_{B} \ln 2, & \mu= \pm t, \\ 0, & \text { otherwise. }\end{cases}
$$

The effect of the localized states at $E= \pm 1$ on the entropy is illustrated in Fig. 1(a).

\section{b. Half-odd integer flux: $\Phi=(n+1 / 2) \phi_{0}$}

For $\Phi=(n+1 / 2) \phi_{0}, g(E)$ is regular except at $E=0, \pm \sqrt{3}$. Evaluating the limiting behavior as $\Phi \rightarrow(n+1 / 2) \phi_{0}$, one finds

$$
\begin{aligned}
g\left(\omega,(n+1 / 2) \phi_{0}\right)= & \frac{\delta(E)}{t}+\frac{\delta(E-\sqrt{3})}{t}+\frac{\delta(E+\sqrt{3})}{t} \\
& +\frac{U}{\pi t} \frac{E^{4}+3}{E^{2}\left(E^{2}-3\right)^{2}+U^{2}\left(E^{2}-1\right)^{2}} .
\end{aligned}
$$

For this case,

$$
\lim _{T \rightarrow 0} S(\mu, T)= \begin{cases}k_{B} \ln 2, & \mu= \pm \sqrt{3} t \\ k_{B} \ln 2, & \mu=0, \\ 0, & \text { otherwise. }\end{cases}
$$

\section{REFERENCES}

${ }^{1}$ A. Levy, R. Alicki, and R. Kosloff, Phys. Rev. E 85, 061126 (2012).

${ }^{2}$ M. Kolár, D. Gelbwaser-Klimovsky, R. Alicki, and G. Kurizki, Phys. Rev. Lett. 109, 090601 (2012).

${ }^{3}$ B. Cleuren, B. Rutten, and C. Van den Broeck, Phys. Rev. Lett. 108, 120603 (2012).

${ }^{4}$ A. Levy, R. Alicki, and R. Kosloff, Phys. Rev. Lett. 109, 248901 (2012).

${ }^{5}$ R. Kosloff, Entropy 15, 2100 (2013).

${ }^{6}$ L. Masanes and J. Oppenheim, Nat. Commun. 8, 14538 (2017).

${ }^{7}$ G. W. Ford and R. F. O'Connell, Physica E 29, 82 (2005).

${ }^{8}$ R. F. O'Connell, J. Stat. Phys. 124, 15 (2006).

${ }^{9}$ T. M. Nieuwenhuizen and A. E. Allahverdyan, Phys. Rev. E 66, 036102 (2002).

${ }^{10}$ M. Esposito, M. A. Ochoa, and M. Galperin, Phys. Rev. B 92, 235440 (2015).

${ }^{11}$ M. F. Ludovico, J. S. Lim, M. Moskalets, L. Arrachea, and D. Sánchez, Phys. Rev, B 89, 161306 (2014).

${ }^{12}$ M. F. Ludovico, L. Arrachea, M. Moskalets, and D. Sánchez, Phys. Rev. B 97, 041416 (2018).

${ }^{13}$ A. Bruch, M. Thomas, S. Viola Kusminskiy, F. von Oppen, and A. Nitzan, Phys. Rev. B 93, 115318 (2016).

${ }^{14}$ J. Friedel, Il Nuovo Cimento 7, 287 (1958) (1955-1965).

${ }^{15}$ A. Bruch, C. Lewenkopf, and F. von Oppen, Phys. Rev. Lett. 120, 107701 (2018).

${ }^{16}$ For clarity of notation, Fock-space operators other than the creation and annihilation operators themselves are written with a hat, while operators in the 1-body Hilbert space are written without a hat.

${ }^{17}$ G. Stefanucci and R. van Leeuwen, Nonequilibrium Many-Body Theory of Quantum Systems: A Modern Introduction (Cambridge University Press, 2013).

${ }^{18}$ J. P. Bergfield and C. A. Stafford, Phys. Rev. B 79, 245125 (2009).

${ }^{19}$ M. G. Reuter and T. Hansen, J. Chem. Phys. 141, 181103 (2014).

${ }^{20}$ P. Sam-ang and M. G. Reuter, New J. Phys. 19, 053002 (2017).

${ }^{21}$ V. Gasparian, T. Christen, and M. Büttiker, Phys. Rev. A 54, 4022 (1996).

${ }^{22}$ T. Gramespacher and M. Büttiker, Phys. Rev. B 56, 13026 (1997).

${ }^{23}$ C. A. Stafford, Phys. Rev. B 93, 245403 (2016).

${ }^{24}$ The grand partition function can be written as $\mathcal{Z}(\mu, T)=e^{-\beta \Omega}$.

${ }^{25}$ C. A. Stafford, D. Baeriswyl, and J. Bürki, Phys. Rev. Lett. 79, 2863 (1997).

${ }^{26}$ C. A. Stafford and A. Shastry, J. Chem. Phys. 146, 092324 (2017).

${ }^{27} \mathrm{See}$ Sec. 5.2 of Ref. 28 for a general discussion of subsystem entropy.

${ }^{28}$ A. S. C. Shastry, "Theory of thermodynamic measurements of quantum systems far from equilibrium," Ph.D. thesis, University of Arizona, 2018, copyrightSFDatabase copyright ProQuest LLC; ProQuest does not claim copyright in the individual underlying works; Last updated July 04, 2018.

${ }^{29}$ A. Shastry and C. A. Stafford (unpublished).

${ }^{30}$ A. Shastry and C. A. Stafford, Phys. Rev. B 92, 245417 (2015).

${ }^{31}$ A. Shastry and C. A. Stafford, Phys. Rev. B 94, 155433 (2016)

${ }^{32}$ H. Ness, Phys. Rev. B 89, 045409 (2014).

${ }^{33}$ Y. Dubi and M. Di Ventra, Nano Lett. 9, 97 (2009).

${ }^{34}$ P. A. Jacquet and C.-A. Pillet, Phys. Rev. B 85, 125120 (2012).

${ }^{35}$ J. P. Bergfield, S. M. Story, R. C. Stafford, and C. A. Stafford, ACS Nano 7, 4429 (2013).

${ }^{36}$ J. Meair, J. P. Bergfield, C. A. Stafford, and P. Jacquod, Phys. Rev. B 90, 035407 (2014).

${ }^{37}$ J. P. Bergfield, M. A. Ratner, C. A. Stafford, and M. Di Ventra, Phys. Rev. B 91, 125407 (2015).

${ }^{38}$ M. Büttiker, Phys. Rev. B 40, 3409 (1989).

${ }^{39}$ J. P. Bergfield and C. A. Stafford, Phys. Rev. B 90, 235438 (2014).

${ }^{40} \mathrm{H}$. Haug and A.-P. Jauho, Quantum Kinetics in Transport and Optics of Semiconductors, Solid-State Sciences Vol. 123 (Springer, 1996).

${ }^{41} \mathrm{~J}$. C. Cuevas and E. Scheer, Molecular Electronics (World Scientific, 2010), https://www.worldscientific.com/doi/pdf/10.1142/7434.

${ }^{42}$ J. D. Barr, C. A. Stafford, and J. P. Bergfield, Phys. Rev. B 86, 115403 (2012). 